\title{
Predictors of early recurrence following a curative resection in patients with a carcinoma of the ampulla of Vater
}

\author{
Chung Hyeun Ma'1, Jae Hoon Lee², Ki Byung Song'², Dae Wook Hwang², Song Cheol Kim² \\ ${ }^{1}$ Department of Surgery, Gangneung Asan Hospital, University of Ulsan College of Medicine, Gangneung, Korea \\ 2Division of Hepatobiliary and Pancreatic Surgery, Department of Surgery, Asan Medical Center, University of Ulsan College of \\ Medicine, Seoul, Korea
}

\begin{abstract}
Purpose: Ampulla of Vater (AoV) carcinoma has a relatively good prognosis. The 5 -year recurrence rate for AoV is still around $40 \%-50 \%$ however, and most recurrences occur in the early period. The aim of this study was to identify predictors of an early recurrence in AoV patients following a curative resection.

Methods: The clinicopathological data for 501 consecutive patients that underwent a resection for AoV in our institute between January 2000 and December 2015 were retrospectively reviewed. The characteristics of any recurrences and early recurrence patients were analyzed accordingly. Early recurrence was defined as occurring within one year of resection.

Results: There were 170 diagnosed recurrences in our study population, $57.1 \%$ of whom were men, with a mean age of 60.1 years (range, 30-94 years). Almost all of the study patients underwent a pancreaticoduodenectomy, and $9 \%$ underwent minimally invasive surgery. Of the 170 recurrent cases, 107 were diagnosed with an early recurrence and had 1-, 3-, and 5-year overall survival rates of $77.7 \%, 18.4 \%, 10.5 \%$, respectively. The factors that significantly influenced early recurrences, determined by multivariate analysis, lymphovascular invasion (LVI), lymph node ratio (LNR), and poor differentiation were found to be independent determinants of a recurrence within 1 year.

Conclusion: An early recurrence in AoV patients is ultimately lethal even though this cancer has a good prognosis. LVI, $L N R$, and poor differentiation are powerful predictors of an early recurrence in AoV. Hence, intensive surveillance and new therapeutic strategies should be considered for AoV patients with these predictors following a curative resection.

[Ann Surg Treat Res 2020;99(5):259-267]
\end{abstract}

Key Words: Ampulla of Vater, Early recurrence, Pancreaticoduodenectomy, Periampullary cancer

\section{INTRODUCTION}

The types of cancer that can occur around the ampulla of Vater (AoV) include pancreatic head cancer, distal biliary cancer, and duodenal cancer. AoV cancers typically have a better prognosis than other periampullary malignancies, show a high rate of surgical treatment success, and have a low recurrence rate. Previous studies have reported that $80 \%$ of AoV cancers are resectable at the time of diagnosis [1-5], and that the 5-year survival rate ranges from 30\% to $68 \%$ after surgical treatment, which are far better outcomes than in other periampullary cancers [3,6]. Nevertheless, recurrence is a major cause of mortality from AoV lesions and thus has a significant negative effect on survival outcomes. Although the effects of early recurrence on postoperative mortality have been well studied in colorectal, liver, and pancreatic cancers, there are few
Received March 18, 2020, Revised July 13, 2020, Accepted July 29, 2020

Corresponding Author: Song Cheol Kim

Division of Hepatobiliary and Pancreatic Surgery, Department of Surgery, Asan Medical Center, University of Ulsan College of Medicine, 88 Olympicro 43-gil, Songpa-gu, Seoul 05505, Korea

Tel: +82-2-3010-1521, Fax: +82-2-3010-6701

E-mail: drksc@amc.seoul.kr

ORCID: https://orcid.org/0000-0003-4552-4169

\section{Copyright (c) 2020, the Korean Surgical Society}

(c) Annals of Surgical Treatment and Research is an Open Access Journal. All articles are distributed under the terms of the Creative Commons Attribution NonCommercial License (http://creativecommons.org/licenses/by-nc/4.0/) which permits unrestricted non-commercial use, distribution, and reproduction in any medium, provided the original work is properly cited. 
analyses of its impacts in AoV patients [6-9].

The recurrence rate in patients with AoV cancers has been reported to range from $28 \%$ to $44 \%$ and is manifested in the form of liver metastases, local recurrences, and lymph node recurrence. The capacity of various chemotherapy and radiotherapy protocols to reduce postoperative recurrences in AoV has been evaluated in a number of reports but the findings have been somewhat controversial and no adjuvant therapy has yet been established [3,10-13]. The analysis of factors that may cause an AoV recurrence may provide criteria for adjuvant chemotherapy regimens that can improve survival outcomes following curative resection. However, the incidence of AoV cancer is very low (about $0.2 \%$ of all gastrointestinal cancers) and most prior studies have attempted to predict the prognosis of these cases by analyzing the clinicopathological characteristics of fewer than 100 patients. Indeed, there have been few studies on the factors affecting AoV recurrence [1,2,1421]. The purpose of our present study was to identify the factors affecting the recurrence rate after curative surgery for AoV cancer, particularly in relation to early recurrence, and to thereby provide a basis for establishing novel adjuvant therapy strategies.

\section{METHODS}

\section{Study cohort and end points}

The medical records of $536 \mathrm{AoV}$ cancer patients who received curative resection between January 2000 and November 2015 at the Department of Hepatobiliary and Pancreatic Surgery, Asan Medical Center were reviewed retrospectively. AoV cancer is defined as a malignant neoplasm originating from the ampulla or papilla of duodenum but only histologically diagnosed adenocarcinomas were reviewed. Sixteen cases that had been histopathologically defined as distal biliary, pancreatic, and duodenal cancer were excluded. Eight patients who were diagnosed with double primary cancer were also excluded. Among the remaining cases for study inclusion, 2 patients died due to underlying disease after surgery. The causes of death were an acute myocardial infarction and an acute cerebral infarction. These 2 patients were thus excluded. Nine further patients who underwent follow-ups at other hospitals were also excluded leaving a final study cohort of 501 patients (Fig. 1).

All surgeries were consecutively performed by 5 surgeons who received the same training at Asan Medical Center. A pancreaticoduodenectomy, pylorus-preserving pancreaticoduodenectomy, or laparoscopic pylorus-preserving pancreaticoduodenectomy was performed at the discretion of the surgeon. The tissue samples obtained after surgery were reviewed by 2 histopathologists and classified in accordance with the Cancer Staging Manual of American Joint Committee on Cancer, 7th edition.

Patients attended the outpatient clinic at 3-month intervals during the first 2 years after surgery and at 6-month intervals every 2 years thereafter. Abdominal CT, assays for tumor markers (CEA, CA 19-9), and hematology tests were performed at each follow-up visit. Overall survival and recurrences were determined from the day of surgery. In case of newly developed lesions in abdominal CT or elevated tumor markers, recurrence was suspected. PET-CT was performed to increase the accuracy of detection. The locoregional recurrence was defined as a

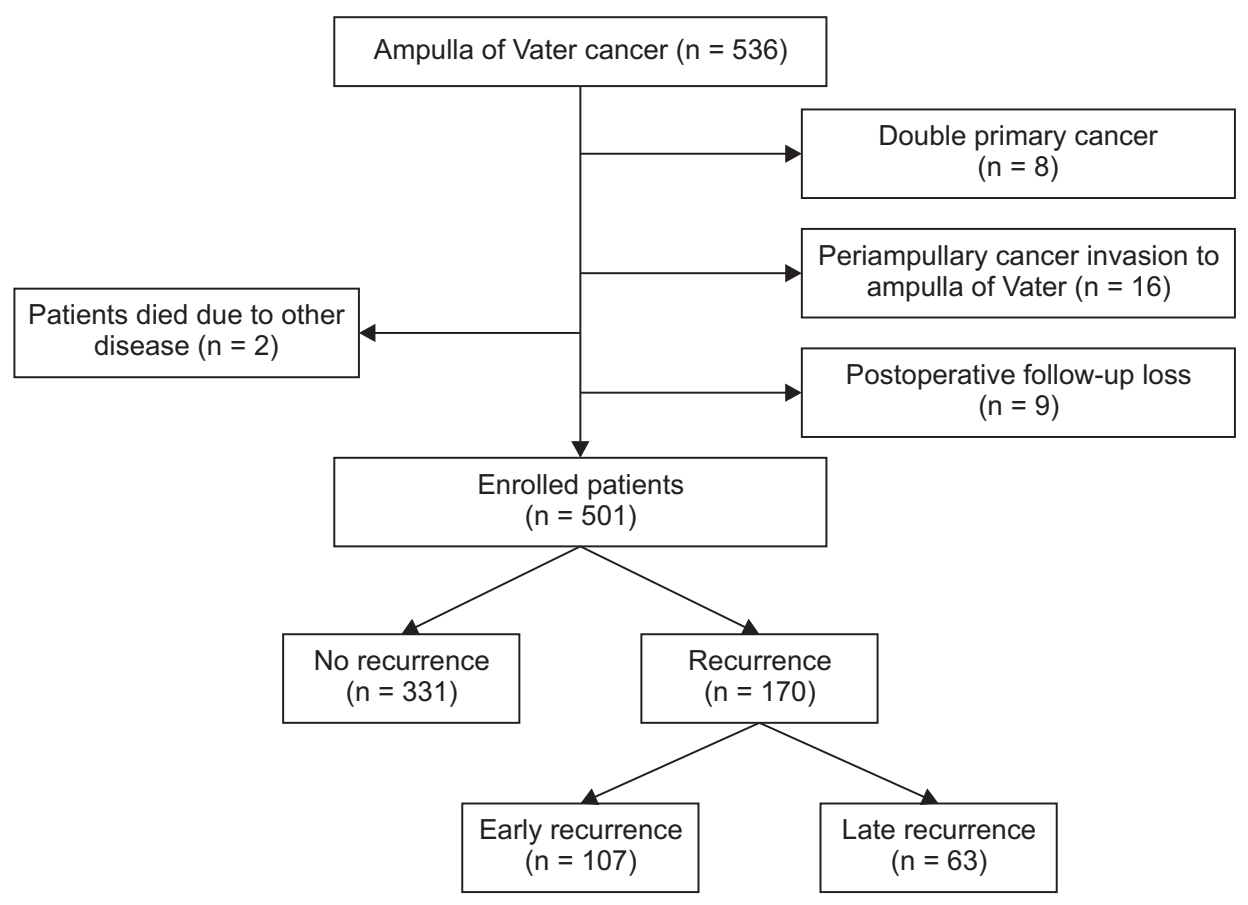

Fig. 1. Flow diagram of patient selection. 
newly developed lesion at the anastomosis site, liver hilum, and lymph node around previous surgical area including superior mesenteric vein root. Distant recurrences were defined as those arising at other organs. Recurrences were confirmed by biopsy when possible. The mean duration to recurrence was 12.28 months in our current study series. An 'early recurrence' was therefore defined as within 12 months of surgery in the present analyses. The primary study endpoint was recurrence-free survival and the secondary endpoint was overall survival.

This retrospective study was approved by the Asan Medical Center Institutional Review Board (No. S2019-22690001). Patient informed consent was waived because of the retrospective nature of the study. Data collection and analysis were performed according to the institutional guidelines, which conformed to the ethical standards of the Declaration of Helsinki.

\section{Statistical analysis}

All statistical analyses were performed using IBM SPSS Statistics software for Windows, ver. 19.0 (IBM Corp., Armonk, NY, USA). Categorical variables were compared using a chisquare test or Fisher exact test in univariate analysis and continuous variables using a t-test, Mann-Whitney test, or analysis of variance. Kaplan-Meier survival analysis was used to determine the survival and recurrence rates. Statistical significance was compared using a log-rank test. Multivariate analysis was conducted using the Cox proportional hazards model to analyze factors affecting early recurrence. A P-value of $<0.05$ was considered statistically significant.

\section{RESULTS}

\section{Characteristics of the recurrence and non- recurrence groups}

A total of 501 patients were included in our current study cohort. Of these cases, 267 (53.3\%) were male, and 234 (46.7\%) were female. The mean age was 61.3 years, and the mean \pm standard deviation of body mass index (BMI) was $23.3 \pm 2.9$ $\mathrm{kg} / \mathrm{m}^{2}$. Before surgery, 152 of these AoV cancer patients (30.3\%) underwent an external drainage procedure such as percutaneous transhepatic biliary drainage, endoscopic retrograde biliary drainage, or endoscopic nasobiliary drainage. In accordance with the surgeon's judgment regarding the extent of disease invasion, a total of 449 of the study patients (89.6\%) underwent open surgery including pancreaticoduodenectomy, pyloruspreserving pancreaticoduodenectomy, and total pancreatectomy. Seven patients (1.4\%) underwent a transduodenal ampullectomy and 45 (9.0\%) underwent a laparoscopic pylorus-preserving pancreaticoduodenectomy. Usually, total pancreatectomy was not performed in patients with AoV cancer, but we had 2 cases. One case was a patient with intraductal papillary mucinous neoplasm of main duct type and high-grade dysplasia in the resection margin. The other case was a patient who had suspected lymphoma in the spleen in the preoperative examination, and the patient required spleen resection. During the operation, 63 patients received transfusions with a mean transfusion volume of 0.8 units ( \pm 2.3 unit). From the pathologic reports, the mean tumor size was determined to be $2.2 \mathrm{~cm} \mathrm{(} \pm$ $1.1 \mathrm{~cm})$ and 151 patients (30.1\%) were lymph node-positive. The histologic grade was diagnosed as undifferentiated cancer in 38

Table 1. Demographics, preoperative data, and operative findings in the study patients with ampulla of Vater cancer who underwent a curative resection $(n=501)$

\begin{tabular}{lccc}
\hline \multicolumn{1}{c}{ Variable } & Non-recurrence group $(\mathrm{n}=331)$ & Recurrence group $(\mathrm{n}=170)$ & P-value $^{\mathrm{a})}$ \\
\hline Age $(\mathrm{yr})$ & $61.9 \pm 9.7$ & $60.1 \pm 9.8$ & 0.045 \\
Sex & & & \\
Male & $169(51.1)$ & $73(57.1)$ & 0.252 \\
Female & $162(48.9)$ & $22.9 \pm 3.3$ & 0.199 \\
Body mass index $\left(\mathrm{kg} / \mathrm{m}^{2}\right)$ & $23.4 \pm 2.7$ & $2.5(0.3-323.6)$ & 0.043 \\
Preoperative CEA $(\mathrm{ng} / \mathrm{mL})$ & $1.70(0.1-8)$ & $32.2(0.6-5470.0)$ & 0.001 \\
Preoperative CA $19-9(\mathrm{U} / \mathrm{mL})$ & $14.9(0.6-981.2)$ & $1.5(0.2-13.6)$ & 0.001 \\
Preoperative bilirubin $(\mathrm{mg} / \mathrm{dL})$ & $0.9(0.2-15.6)$ & $401.3 \pm 92.8$ & 0.536 \\
Operation time $(\mathrm{min})$ & $378.1 \pm 94.6$ & $158(92.9)$ & $12(7.1)$ \\
Operative method & $298(90.0)$ & $1.0 \pm 2.0$ & 0.196 \\
Oppen & $33(10.0)$ & $38(22.4)$ & 0.001 \\
Laparoscopic or robotic MIS & $0.7 \pm 1.8$ & & \\
Transfusion (unit) & $32(9.6)$ & & \\
Adjuvant chemotherapy, yes & & & \\
\hline
\end{tabular}

Values are presented as mean \pm standard deviation, number $(\%)$, or median (range).

MIS, minimally invasive surgery.

${ }^{a)}$ Univariate analysis. ${ }^{\text {b) }}$ Pancreaticoduodenectomy, pylorus-preserving pancreaticoduodenectomy, total pancreatectomy, and transduodenalampullectomy. 
Table 2. Histopathologic findings in the study patients with ampulla of Vater cancer who underwent a curative resection (n $=501)$

\begin{tabular}{|c|c|c|c|}
\hline Variable & Non-recurrence group $(\mathrm{n}=331$ ) & Recurrence group $(n=170)$ & P-value ${ }^{a)}$ \\
\hline Size of tumor $(\mathrm{cm})$ & $2.0 \pm 1.0$ & $2.5 \pm 1.3$ & 0.001 \\
\hline T stage & & & 0.001 \\
\hline Tis & $8(2.4)$ & $0(0)$ & \\
\hline $\mathrm{T} 1$ & $108(32.8)$ & $17(10.0)$ & \\
\hline T2 & $133(40.4)$ & $62(36.5)$ & \\
\hline T3 & $71(21.6)$ & $70(41.2)$ & \\
\hline T4 & $9(2.7)$ & 21 (12.4) & \\
\hline $\mathrm{N}$ stage & & & 0.001 \\
\hline No & $262(81.4)$ & $78(46.2)$ & \\
\hline $\mathrm{N} 1$ & $60(18.6)$ & 91 (53.8) & \\
\hline Resected lymph node & $13.8 \pm 8.1$ & $13.0 \pm 8.4$ & 0.298 \\
\hline Positive lymph node & $0.4 \pm 0.9$ & $1.8 \pm 3.3$ & 0.001 \\
\hline Lymph node ratio & & & 0.001 \\
\hline$<0.2$ & $304(95.3)$ & $130(76.5)$ & \\
\hline$\geq 0.2$ & $15(4.7)$ & $40(23.5)$ & \\
\hline Resection margin & & & 0.084 \\
\hline Ro & $314(99.1)$ & $158(96.3)$ & \\
\hline R1 & $3(0.9)$ & $6(3.7)$ & \\
\hline Histologic grade & & & 0.382 \\
\hline Well to moderate & $284(92.8)$ & $144(90.0)$ & \\
\hline Poor & $22(7.2)$ & $16(10.0)$ & \\
\hline Perineural invasion & & & 0.001 \\
\hline No & $275(85.1)$ & $110(66.7)$ & \\
\hline Yes & $48(14.9)$ & 55 (33.3) & \\
\hline Lymphovascular invasion & & & 0.001 \\
\hline No & $236(72.2)$ & $69(41.3)$ & \\
\hline Yes & $91(27.8)$ & $98(58.7)$ & \\
\hline
\end{tabular}

Values are presented as mean \pm standard deviation or number (\%).

a) Univariate analysis.

patients $(7.6 \%)$.

Of the total study cohort of 501 patients, there were 170 cases of recurrence. There were no statistically significant differences between the BMI, operation time, operative method, or transfusion volume between the 2 groups. However, the patient age was significantly higher in the non-recurrence group $(61.9$ \pm 9.7 years), and the preoperative tumor marker expression levels (CEA, CA 19-9) and bilirubin level were higher in the recurrence cases. Adjuvant chemotherapy was more common in the recurrence group (Table 1). The median total resected lymph node value did not show a statistically significant difference between the groups, but the mean tumor size, $\mathrm{T}$ stage, $\mathrm{N}$ stage, median positive lymph node value, positive lymph node ratio (LNR) $\geq 0.2$, presence of perineural invasion (PNI), presence of lymphovascular invasion (LVI) were significantly increased in the recurrence group compared to the non-recurrence group ( $\mathrm{P}$ $<0.05$ ) (Table 2).

\section{Characteristics of the early and late recurrence groups}

The 3-year survival rate was $18.4 \%$ and the 5-year survival rate was $10.5 \%$ in the early recurrence group. The 3-year and 5-year survival rates in the late recurrence group however were $66.5 \%$ and $26.7 \%$, respectively (Fig. 2). Table 3 presents the results from univariate analysis of the demographic characteristics and clinicopathologic features of each group. There were statistically significant differences found in terms of sex, number of benign lymph nodes, percentage of benign lymph nodes, histologic grade, PNI, and LVI $(\mathrm{P}<0.05)$. Postoperative adjuvant chemotherapy was performed in 9 patients (14.3\%) in the late recurrence group and 29 patients $(27.1 \%)$ in the early recurrence group. There was no significant difference in the survival results between the 2 groups, but the survival outcomes for patients receiving chemotherapy were poorer (Fig. 3).

\section{Recurrence patterns between the early and late recurrence groups \\ Local recurrence and distant metastasis showed a statistically}



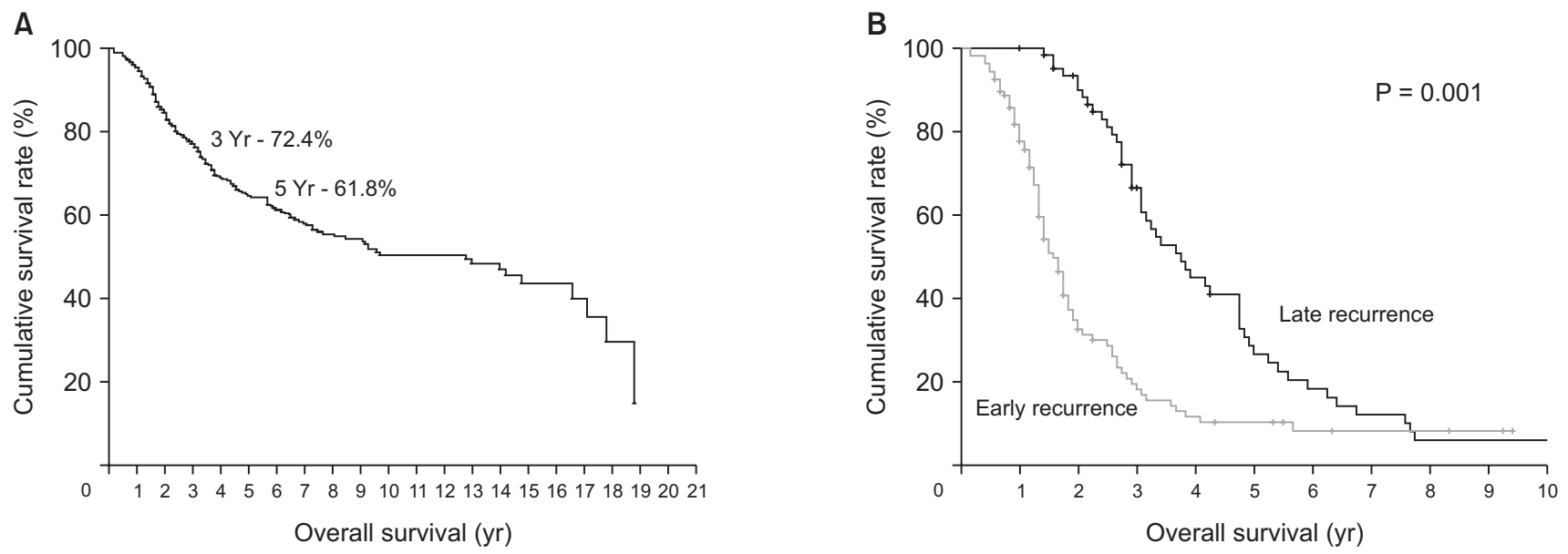

Fig. 2. (A) Kaplan-Meier survival curves after curative resection for ampulla of Vater cancer. (B) Kaplan-Meier survival curves for early and late recurrence patients.

Table 3. Clinicopathological findings in the study patients with ampulla of Vater cancer who underwent curative resection (n $=170)$

\begin{tabular}{|c|c|c|c|}
\hline Variable & Late recurrence group $(n=63)$ & Early recurrence group $(n=107)$ & P-value ${ }^{a)}$ \\
\hline Age (yr) & $60.2 \pm 10.6$ & $60.1 \pm 9.4$ & 0.948 \\
\hline Sex & & & 0.004 \\
\hline Male & $27(42.9)$ & $69(64.5)$ & \\
\hline Female & $36(57.1)$ & $38(35.5)$ & \\
\hline Body mass index $\left(\mathrm{kg} / \mathrm{m}^{2}\right)$ & $22.8 \pm 2.6$ & $22.9 \pm 3.6$ & 0.874 \\
\hline Preoperative CEA (ng/mL) & $2.4(0.5-40.4)$ & $2.5(0.3-323.6)$ & 0.441 \\
\hline Preoperative CA 19-9 (U/mL) & $26.9(1.5-557.5)$ & $35.3(0.6-5,470.0)$ & 0.125 \\
\hline Preoperative bilirubin (mg/dL) & $1.4(0.2-9.6)$ & $1.5(0.2-13.6)$ & 0.441 \\
\hline Operation time (min) & $377.3 \pm 59.5$ & $416.1 \pm 101.7$ & 0.065 \\
\hline Size of tumor $(\mathrm{cm})$ & $2.4 \pm 1.4$ & $2.5 \pm 1.2$ & 0.585 \\
\hline T stage & & & 0.070 \\
\hline T1 & $11(17.5)$ & $6(5.7)$ & \\
\hline $\mathrm{T} 2$ & $24(38.1)$ & $38(35.8)$ & \\
\hline $\mathrm{T} 3$ & $21(33.3)$ & $48(45.3)$ & \\
\hline $\mathrm{T} 4$ & $7(11.1)$ & $14(13.2)$ & \\
\hline $\mathrm{N}$ stage & & & 0.439 \\
\hline $\mathrm{N} 1$ & $32(50.8)$ & $46(43.4)$ & \\
\hline $\mathrm{N} 2$ & $31(49.2)$ & $60(56.6)$ & \\
\hline Positive lymph node & $1.1 \pm 1.7$ & $2.1 \pm 3.9$ & 0.044 \\
\hline Lymph node ratio & & & 0.029 \\
\hline$<0.2$ & $53(85.5)$ & $75(71.4)$ & \\
\hline$\geq 0.2$ & $9(14.5)$ & $30(28.6)$ & \\
\hline Histologic grade & & & 0.001 \\
\hline Well to moderate & $60(100)$ & $81(83.5)$ & \\
\hline Poor & $0(0)$ & $16(16.5)$ & \\
\hline Perineural invasion & & & 0.036 \\
\hline No & $46(76.7)$ & $62(61.4)$ & \\
\hline Yes & $14(23.3)$ & $39(38.6)$ & \\
\hline Lymphovascular invasion & & & 0.001 \\
\hline No & $35(58.3)$ & $33(32.7)$ & \\
\hline Yes & $25(41.7)$ & $68(67.3)$ & \\
\hline Adjuvant chemotherapy, yes & $9(14.3)$ & $29(27.1)$ & 0.053 \\
\hline
\end{tabular}

Values are presented as mean \pm standard deviation, number (\%), or median (range).

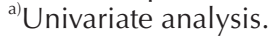



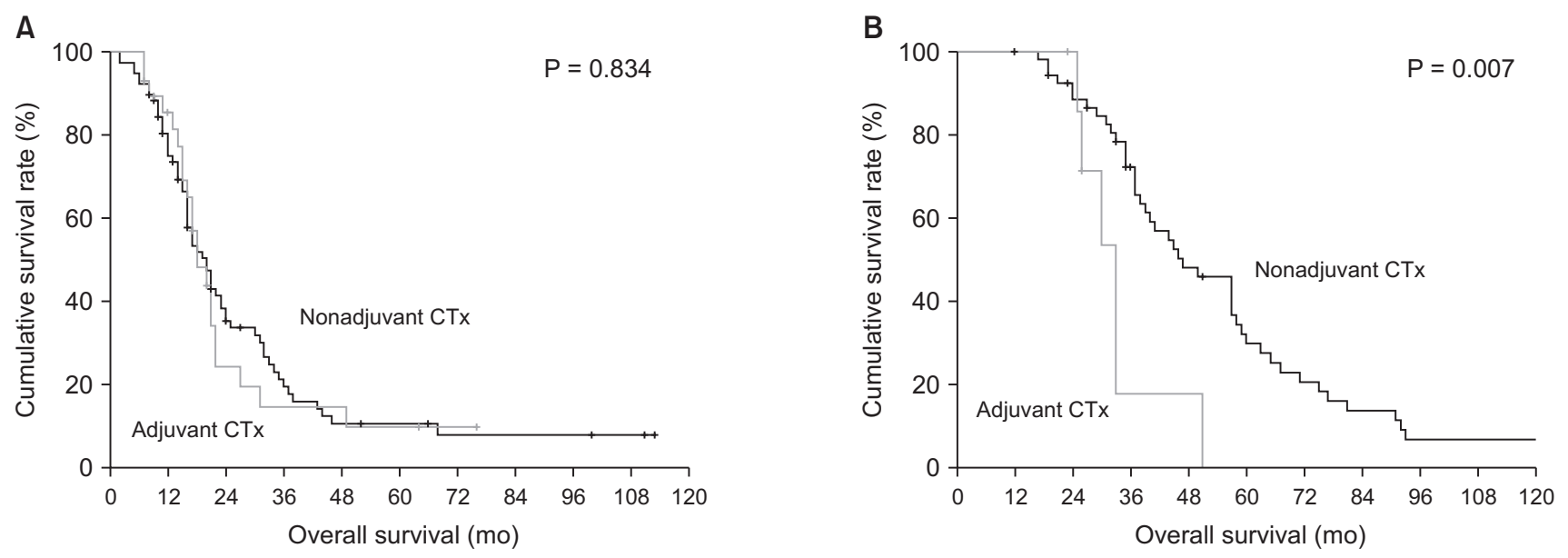

Fig. 3. (A) Overall survival curve with and without adjuvant chemotherapy (CTx) among early recurrence group. (B) Overall survival curve with and without adjuvant CTx among late recurrence group.

Table 4. Comparison of the patterns of recurrence after curative resection in the early and late recurrence groups $(n=170)$

\begin{tabular}{lcc}
\hline \multicolumn{1}{c}{ Recurrence } & Early recurrence group $(\mathrm{n}=107)$ & Late recurrence group $(\mathrm{n}=63)$ \\
\hline Locoregional recurrence & $19(17.8)$ & $22(34.9)$ \\
Distant metastasis & $88(82.2)$ & $41(65.1)$ \\
Locoregional + distant metastasis & $32(29.9)$ & $17(27.0)$ \\
Multi-organ metastasis & $16(15.0)$ & $7(11.1)$ \\
Liver & $30(28.0)$ & $6(9.5)$ \\
Lung & $3(2.8)$ & $4(6.3)$ \\
Peritoneum & $6(5.6)$ & $6(9.5)$ \\
Pancreas & $1(0.9)$ & $3(4.8)$ \\
Bone & $0(0)$ & $1(1.6)$ \\
\hline
\end{tabular}

Values are presented as number (\%).

significant difference between the 2 groups, with distant metastases being far more prominent in the early recurrence group. In subgroup analysis, 32 early recurrence patients (29.9\%) were found to have a local recurrence with distant metastasis. In addition, 30 patients (28.0\%) had a liver metastasis only, and 19 patients $(17.8 \%)$ had a local recurrence only, in the early recurrence group. Two or more other metastases were found in 16 patients (15.0\%). In addition, 6 patients had peritoneal seeding (5.6\%), there were 3 cases of lung metastases (2.8\%), and 1 patient with pancreatic metastasis $(0.9 \%)$. Bone metastases were found in one patient in the late recurrence group (Table 4 , Fig. 4).

\section{Analysis of factors affecting recurrence and early recurrence}

Multivariate analysis was performed to analyze factors affecting recurrence. As a result, advanced T stage (T3/T4), LNR $(\geq 0.2)$, and LVI showed significant differences $(P<0.05)$ (Table 5). In addition, in the multivariate analysis conducted

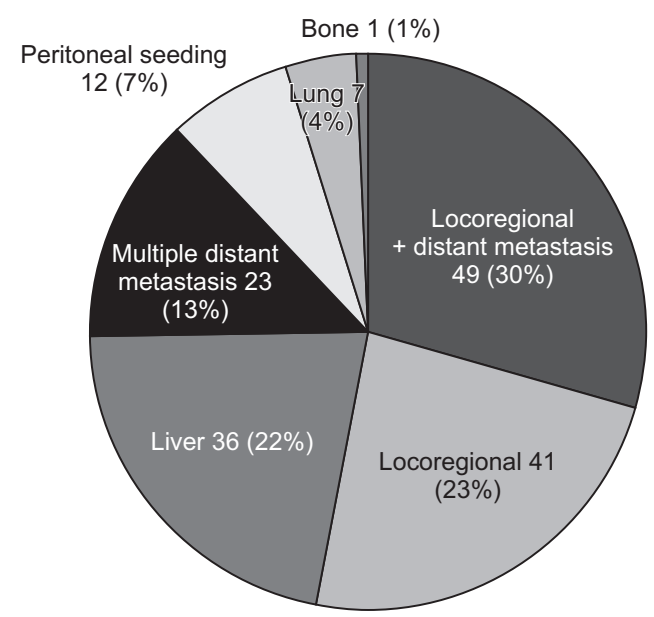

Fig. 4. Patterns of recurrence after curative resection in the 170 recurred patients.

to analyze factors affecting early recurrence, poor histologic type was analyzed as an independent factor influencing early recurrence along with LNR and LVI $(\mathrm{P}<0.05)$ (Table 6). 
Table 5. Cox proportional hazard analysis of recurrence factors in the study patients with ampulla of Vater cancer who underwent curative resection

\begin{tabular}{lccc}
\hline \multirow{2}{*}{\multicolumn{1}{c}{ Characteristic }} & \multicolumn{3}{c}{ Multivariate } \\
\cline { 2 - 5 } & HR & $95 \% \mathrm{Cl}$ & P-value \\
\hline Advanced T stage, T3/T4 & 2.906 & $1.508-5.600$ & 0.001 \\
Lymph node ratio $\geq 0.2$ & 3.112 & $1.589-6.095$ & 0.001 \\
Lymphovascular invasion, yes & 4.061 & $1.929-8.552$ & 0.001 \\
\hline
\end{tabular}

Comparison of the recurrence groups with the non-recurrence group.

$\mathrm{HR}$, hazard ratio; $\mathrm{Cl}$, confidence interval.

\section{DISCUSSION}

The survival rate after a curative resection in AoV cancer is reported to be $30 \%-60 \%$, which is regarded as prognostically favorable among the periampullary cancers. This survival outcome is due to the anatomical structure of AoV lesions. Symptoms of biliary tract obstruction can be observed in the early stages of the disease, so they are often detected early and can be treated with surgery [3]. Notably however, recurrences still arise in AoV cancer patients after surgery. The 5-year survival rate among patients with early recurrence is only $10.5 \%$, which is much lower than that in cases of late recurrence (26.7\%). There have been many studies on the prognostic factors in AoV cancer but few reports on the types of recurrence or factors affecting recurrence in the affected patients. In addition, the incidence of AoV cancer is lower than that of other periampullary cancer types, and the majority of previous studies of AoV tumors have therefore involved less than 100 patients, which reduced their statistical power. The strength of our present study lies in the fact that we enrolled a large cohort of patients that underwent standardized surgical procedures in a single center.

We focused on our current investigation on the analysis of AoV cancer patients with an early recurrence; within 12 months of their resection. This is because more than $60 \%$ of patients who underwent radical resection recurred within 12 months, and the 3-year survival rate of this patient group was $19.5 \%$, and the 5 -year survival rate was $10.5 \%$, which was significantly lower than that of the late recurrence group. In previous studies of AoV cancer recurrences, T stage, local lymph node metastasis, positive LNR, LVI, PNI, and a preoperatively increased CEA have been reported as recurrence factors $[2,6,15,22,23]$. In our present study, the factors affecting recurrence included, positive LNR (LNR $\geq 0.2$ ), LNI, and advanced T stage showed significant results which was consistent with previous findings. We further analyzed early and late recurrence, and found that LNI, positive LNR, and poorly differentiated tumor type were strong independent predictive factors for recurrence (Table 6).
Table 6. Cox proportional hazard analysis of early recurrence factors

\begin{tabular}{lccc}
\hline \multirow{2}{*}{\multicolumn{1}{c}{ Characteristic }} & \multicolumn{3}{c}{ Multivariate } \\
\cline { 2 - 4 } & HR & $95 \% \mathrm{Cl}$ & P-value \\
\hline Lymphovascular invasion, yes & 3.459 & $2.225-5.377$ & 0.001 \\
Lymph node ratio $\geq 0.2$ & 2.916 & $1.855-4.582$ & 0.001 \\
Histologic type, poor & 2.161 & $1.261-3.701$ & 0.005 \\
\hline
\end{tabular}

$\mathrm{HR}$, hazard ratio; $\mathrm{Cl}$, confidence interval.

LVI and a positive LNR were notable in particular for showing an association with recurrence in both groups.

Howe et al. [24] previously reported a mean survival time of 23.6 months in AoV cancer patients with lymph node involvement, which was significantly lower than the 67.7 months in patients without lymph node involvement. Other studies have reported a 5 -year survival rate of $0 \%-30 \%$ for AoV cancer patients with lymph node involvement, but a good survival rate of up to $78 \%$ for cases without lymph node involvement [3,25]. However, in our study multivariate analysis of AoV cancer recurrence indicated that the presence of lymph node involvement was not significant. In our current study series, the positive LNR was analyzed as a predictive factor for recurrence and early recurrence, but the prognostic effect of this was controversial. Sakata et al. [25] reported previously that positive LNR is a better predictor of prognosis than a positive or negative lymph node involvement. In a prior study conducted by Hurtuk et al. [26], a positive LNR was analyzed via 4 subgroups; $\mathrm{LNR}=0, \mathrm{LNR} \leq 0.2$, LNR $\leq 0.4$, and LNR $>0.4$. These authors reported a higher positive lymph node rate was associated with a poorer prognosis. In our present study, a positive LNR was analyzed as an independent factor for early and late recurrence and was found to be a strong prognostic indicator.

The most frequent recurrence patterns in $\mathrm{AoV}$ cancer are liver metastasis $(53 \%-60 \%)$ followed by a distant lymph node metastasis $(28 \%-60 \%)[4,18,27]$. In our current study, the recurrence location was analyzed by stratification with the period of recurrence. Early recurrence was high in liver metastases (28.0\%) and multi-organ metastases (14.0\%), and in cases where distant metastases occurred simultaneously with local recurrence (28.8\%). Through this, $82.2 \%$ of our study patients with an early recurrence showed a distant metastasis.

Local recurrences made up $23 \%$ of all recurrences in our patient population, with most arising in the mesenteric and paraaortic lymph nodes. Lee et al. [28] reported that local recurrence in AoV cancer occurred frequently in the superior mesenteric lymph nodes and posterior pancreaticoduodenal lymph nodes, and suggested that extended lymph node dissection should be performed in patients with this recurrence. In contrast, Yeo et al. [29] conducted randomized controlled 
trials of extended lymph node dissection of periampullary cancer and reported no significant differences in the survival rate between a standard lymph node dissection and extended lymph node dissection group. That study was limited however by the fact that it could not properly analyze the characteristics of AoV cancer as both pancreatic cancer and distal biliary cancer patients were included, with different biological characteristics. There for a randomized controlled study of a superior mesenteric lymph node and paraaortic lymph node dissection in AoV cancer patients only would thus be desirable. We suggest that considering that a high rate of local recurrence (23\%) and a positive LNR are important recurrence factors, a superior mesenteric lymph node and paraaortic lymph node dissection may reduce the incidence of local recurrences.

Since local recurrences and distant metastasis in AoV cancer have often been found to occur at the same time, there have been many cases involving concurrent chemoradiotherapy after curative resection. However, recent reports from Zhou et al. [10] have indicated that adjuvant chemotherapy after a radical resection did not improve the survival rate and there is still controversy about the use of postoperative adjuvant chemotherapy as there is no standard protocol. In our present analyses, the survival rate was not improved by postoperative adjuvant chemotherapy. Notably however, we included patients who had been diagnosed after 2000 and our results may need to be interpreted with some caution as chemotherapy guidelines have changed several times over the 15 years of our study period. Recently, Cloyd et al. [30] conducted a study of neoadjuvant chemotherapy impacts in AoV cancer patients. These authors found no significant improvement in survival but did note that the survival rate of patients who underwent curative resection after neoadjuvant chemotherapy was better than previously reported.

Our current findings revealed that early recurrence negatively impacts survival in AoV cancer patients and we identified some of the predictive factors for this. This may help future development of various treatment management approaches after a curative resection of $\mathrm{AoV}$ cancer. In the future, studies on correctable factors preoperatively that affect early recurrence of AoV cancer will be needed.
In conclusion, an early recurrence following a surgical resection for AoV cancer leads to a poorer survival outcome compared to late recurrence. Factors that may increase the risk of an early recurrence include LVI, a positive LNR, and a poorly differentiated tumor type. Most of the recurred patients in our present cohort had a distant metastasis, many of which were associated with a local recurrence. Our findings provide important information for the future development of new treatment methods after the primary surgical intervention in AoV cancer patients. Based on this, it is considered that active research on adjuvant chemotherapy performed after surgery is needed to improve the survival rate of patients who early recurrence.

\section{ACKNOWLEDGEMENTS}

The authors would like to thank Young Hun You, M.D. in Department of Surgery, Samsung Medical Center, Sungkyunkwan University School of Medicine for help with data analysis.

\section{Conflicts of Interest}

No potential conflict of interest relevant to this article was reported.

\section{ORCID iD}

Chung Hyeun Ma: https://orcid.org/0000-0002-7005-1079

Jae Hoon Lee: https://orcid.org/0000-0002-6170-8729

Ki Byung Song: https://orcid.org/0000-0001-5422-5481

Dae Wook Hwang: https://orcid.org/0000-0002-1749-038X

Song Cheol Kim: https://orcid.org/0000-0003-4552-4169

\section{Author Contribution}

Conceptualization: CHM, SCK

Formal Analysis: KBS

Investigation: JHL, DWH

Methodology: CHM

Project Administration: CHM, SCK

Writing - Original Draft: CHM

Writing - Review \& Editing: SCK, DWH, KBS, JHL

\section{REFERENCES}

1. Bucher P, Chassot G, Durmishi Y, Ris F, Morel P. Long-term results of surgical treatment of Vater's ampulla neoplasms. Hepatogastroenterology 2007:54:1239-42.

2. Park JS, Yoon DS, Kim KS, Choi JS, Lee
WJ, Chi HS, et al. Factors influencing recurrence after curative resection for ampulla of Vater carcinoma. J Surg Oncol 2007:95:286-90.

3. Bhatia S, Miller RC, Haddock MG,
Donohue JH, Krishnan S. Adjuvant therapy for ampullary carcinomas: the Mayo Clinic experience. Int J Radiat Oncol Biol Phys 2006;66:514-9.

4. Todoroki T, Koike N, Morishita Y, 
Kawamoto T, Ohkohchi N, Shoda J, et al. Patterns and predictors of failure after curative resections of carcinoma of the ampulla of Vater. Ann Surg Oncol 2003;10:1176-83.

5. Beger HG, Treitschke F, Gansauge F, Harada N, Hiki N, Mattfeldt T. Tumor of the ampulla of Vater: experience with local or radical resection in 171 consecutively treated patients. Arch Surg 1999;134:526-32.

6. Hsu HP, Shan YS, Hsieh YH, Yang TM, Lin PW. Predictors of recurrence after pancreaticoduodenectomy in ampullary cancer: comparison between non-, early and late recurrence. J Formos Med Assoc 2007;106:432-43.

7. Huh JW, Kim CH, Lim SW, Kim HR, Kim YJ. Early recurrence in patients undergoing curative surgery for colorectal cancer: is it a predictor for poor overall survival? Int J Colorectal Dis 2013;28:11439.

8. Yamamoto Y, Ikoma H, Morimura R, Konishi H, Murayama Y, Komatsu S, et al. Optimal duration of the early and late recurrence of pancreatic cancer after pancreatectomy based on the difference in the prognosis. Pancreatology 2014:14:524-9.

9. Cheng Z, Yang P, Qu S, Zhou J, Yang J, Yang $\mathrm{X}$, et al. Risk factors and management for early and late intrahepatic recurrence of solitary hepatocellular carcinoma after curative resection. HPB (Oxford) 2015;17:422-7.

10. Zhou J, Hsu CC, Winter JM, Pawlik TM, Laheru D, Hughes MA, et al. Adjuvant chemoradiation versus surgery alone for adenocarcinoma of the ampulla of Vater. Radiother Oncol 2009;92:244-8.

11. Lee JH, Whittington R, Williams NN, Berry MF, Vaughn DJ, Haller DG, et al. Outcome of pancreaticoduodenectomy and impact of adjuvant therapy for ampullary carcinomas. Int J Radiat Oncol Biol Phys 2000;47:945-53.

12. Krishnan S, Rana V, Evans DB, Varadhachary G, Das P, Bhatia S, et al. Role of adjuvant chemoradiation therapy in adenocarcinomas of the ampulla of vater.
Int J Radiat Oncol Biol Phys 2008;70:73543.

13. Kwon J, Kim BH, Kim K, Chie EK, Ha SW. Survival benefit of adjuvant chemoradiotherapy in patients with ampulla of Vater cancer: a systematic review and meta-analysis. Ann Surg 2015:262:47-52.

14. Talamini MA, Moesinger RC, Pitt HA, Sohn TA, Hruban RH, Lillemoe KD, et al. Adenocarcinoma of the ampulla of Vater: a 28-year experience. Ann Surg 1997:225:590-600.

15. Kim KJ, Choi DW, Kim WS, Kim MJ, Song SC, Heo JS, et al. Adenocarcinoma of the ampulla of Vater: predictors of survival and recurrence after curative radical resection. Korean J Hepatobiliary Pancreat Surg 2011;15:171-8.

16. Qiao QL, Zhao YG, Ye ML, Yang YM, Zhao JX, Huang YT, et al. Carcinoma of the ampulla of Vater: factors influencing long-term survival of 127 patients with resection. World J Surg 2007:31:137-46.

17. Hsu HP, Yang TM, Hsieh YH, Shan YS, Lin PW. Predictors for patterns of failure after pancreaticoduodenectomy in ampullary cancer. Ann Surg Oncol 2007;14:50-60.

18. Kim RD, Kundhal PS, McGilvray ID, Cattral MS, Taylor B, Langer B, et al. Predictors of failure after pancreaticoduodenectomy for ampullary carcinoma. J Am Coll Surg 2006;202:112-9.

19. Allema JH, Reinders ME, van Gulik TM, van Leeuwen DJ, Verbeek PC, de Wit LT, et al. Results of pancreaticoduodenectomy for ampullary carcinoma and analysis of prognostic factors for survival. Surgery 1995;117:247-53.

20. Choi SB, Kim WB, Song TJ, Suh SO, Kim YC, Choi SY. Surgical outcomes and prognostic factors for ampulla of Vater cancer. Scand J Surg 2011;100:92-8.

21. Carter JT, Grenert JP, Rubenstein L, Stewart L, Way LW. Tumors of the ampulla of vater: histopathologic classification and predictors of survival. J Am Coll Surg 2008:207:210-8.

22. Woo SM, Ryu JK, Lee SH, Yoo JW, Park JK, Kim YT, et al. Recurrence and prognostic factors of ampullary carcinoma after radical resection: comparison with distal extrahepatic cholangiocarcinoma. Ann Surg Oncol 2007;14:3195-201.

23. Colussi O, Voron T, Pozet A, Hammel P, Sauvanet A, Bachet JB, et al. Prognostic score for recurrence after Whipple's pancreaticoduodenectomy for ampullary carcinomas; results of an AGEO retrospective multicenter cohort. Eur J Surg Oncol 2015;41:520-6.

24. Howe JR, Klimstra DS, Moccia RD, Conlon KC, Brennan MF. Factors predictive of survival in ampullary carcinoma. Ann Surg 1998:228:87-94.

25. Sakata J, Shirai Y, Wakai T, Ajioka Y, Akazawa K, Hatakeyama K. Assessment of the nodal status in ampullary carcinoma: the number of positive lymph nodes versus the lymph node ratio. World J Surg 2011:35:2118-24.

26. Hurtuk MG, Hughes C, Shoup M, Aranha GV. Does lymph node ratio impact survival in resected periampullary malignancies? Am J Surg 2009;197:348-52.

27. Kayahara M, Nagakawa T, Ohta T, Kitagawa H, Miyazaki I. Surgical strategy for carcinoma of the papilla of Vater on the basis of lymphatic spread and mode of recurrence. Surgery 1997;121:611-7.

28. Lee JH, Lee KG, Ha TK, Jun YJ, Paik SS, Park HK, et al. Pattern analysis of lymph node metastasis and the prognostic importance of number of metastatic nodes in ampullary adenocarcinoma. Am Surg 2011;77:322-9.

29. Yeo CJ, Cameron JL, Lillemoe KD, Sohn TA, Campbell KA, Sauter PK, et al. Pancreaticoduodenectomy with or without distal gastrectomy and extended retroperitoneal lymphadenectomy for periampullary adenocarcinoma, part 2: randomized controlled trial evaluating survival, morbidity, and mortality. Ann Surg 2002;236:355-68.

30. Cloyd JM, Wang H, Overman M, Zhao J, Denbo J, Prakash L, et al. Influence of preoperative therapy on short- and long-term outcomes of patients with adenocarcinoma of the ampulla of Vater. Ann Surg Oncol 2017;24:2031-9. 